\title{
A NOTE ON AGGREGATION OF CES-TYPE PRODUCTION FUNCTIONS
}

\author{
S. SCHIM van der LOEFF and R. HARKEMA* \\ Econometric Institute, Erasmus University, Rotterdam, The Netherlands \\ Received February, 197 !
}

In this note we shall be concerned with the aggregation of the constant elasticity of substitution (CES) type of production function. In particuiar will derive the error made by using the arithmetic averages as they are usually published, rather than the theoretically required averages.

Let us consider firms $i(i=1, \ldots, N)$ producing according to a CES production function schedule, as follows

$$
\begin{aligned}
V_{l t}=\gamma\left[\delta K_{l t}^{-\rho}+(1-\delta) L_{t t}^{-\rho}\right]^{-v / \rho}, \quad i & =1, \ldots, N ; \quad t=1, \ldots, T, \\
v & >0, \quad \gamma>0, \\
& -1<\rho<\infty, \quad 0 \leqslant \delta \leqslant 1,
\end{aligned}
$$

where $V_{i t}, K_{i t}$ and $L_{i t}$ are measures of value added, capital services and labour services of firm $i$ at time $t$, respectively. Rewriting (1), we have

$$
\gamma_{i t}^{-\rho / \nu}=\gamma^{-\rho / v} \delta K_{i t}^{-\rho}+\gamma^{-\rho / \nu}(1-\delta) L_{i t}^{-\rho} \text {. }
$$

Adding over the $N$ firms, we obtain

$$
\sum_{i=1}^{N} V_{i}^{-\rho / \nu}=y^{-\rho / v} \delta \sum_{i=1}^{N} K_{i t}^{-\rho}+\gamma^{-\rho / \gamma}(1-\delta) \sum_{i=1}^{N} L_{i t}^{-\rho} .
$$

We now want to specify our aggregate (macro-) model by analogy to the micromodel (1) or (2). Denoting the aggregates of $V_{t t}, K_{t t}$, and $L_{t t}$ by $V_{t}, K_{t}$, and $L_{t}$, respectively, we thus desire

$$
V_{t}^{-\rho / v}=\gamma^{-\rho / v} \delta K_{t}^{-\rho}+y^{-\rho / v}(1-\delta) L_{t}^{-\rho} .
$$

-The authors are indebted to Professor T. Kloek for some valuable comments. 
This leads us to formulace ous aggregates in the following form:

$$
\begin{aligned}
& V_{t}=\left(\sum_{i=1}^{N} V_{i t}^{-\rho / v}\right)^{-v / \rho}, \\
& K_{t}=\left(\sum_{i=1}^{N} K_{i t}^{-\rho}\right)^{-1 / \rho}, \\
& L_{t}=\left(\sum_{i=1}^{N} L_{i t}^{-\rho}\right)^{-1 / p} .
\end{aligned}
$$

Let us now consider the error made by approximating the theoretically desired mean,

$$
M=\left(\frac{1}{N} \sum_{i=1}^{N} x_{i}^{i}\right)^{1 / r},
$$

by the usually published arithmetic mean. The latter is defined as ${ }^{1}$

$$
A=\frac{1}{N} \sum_{i=1}^{N} x_{i},
$$

and may be obtained as a special case of $(6)$ by substituting $r=1$. We denote another limiting case of (6), namely the case of $r \rightarrow 0,{ }^{2}$ the geometric nean by

$$
G=\left(\prod_{i=1}^{N} x_{i}\right)^{1 / N}
$$

Let us rewrite (6) as follows: ${ }^{3}$

$$
f(r)=\ln Z(r)-\ln \dot{N},
$$

where

$$
f(r)=r \ln M
$$

and

$$
Z(r)=\sum_{i=1}^{N} x_{i}^{r} .
$$

'It should be noted that it is immaterial for our further derivations whether we use weights $w_{i}(l=1, \ldots, N) ; \Sigma_{l=1}^{N} w_{l}=1 ; w_{1} \geqslant 0$, or not.

2See Hardy, Littlewood and Polya (1967, p. 15).

'The fellowing derivations mainly follow along a line given by Bridze (1971) in connection with CES production functions. 
We have

$$
\begin{aligned}
& f^{\prime}(r)=Z^{\prime}(r)[Z(r)]^{-1} \\
& f^{\prime \prime}(r)=Z^{\prime \prime}(r)[Z(r)]^{-1}-\left[Z^{\prime}(r)\right]^{2}[Z(r)]^{-2} \\
& f^{\prime \prime}(r)=Z^{\prime \prime}(r)[Z(r)]^{-1}-3 Z^{\prime \prime}(r) Z^{\prime}(r)[Z(r)]^{-2}+2\left[Z^{\prime}(r)\right]^{3}[Z(r)]^{-3}
\end{aligned}
$$

We further know,

$$
Z^{(k)}(r)=\sum_{i=1}^{N} x_{i}^{f}\left(\ln x_{i}\right)^{k}
$$

where $Z^{(k)}(r)$ denotes the $k$ th derivative of $Z(r)$ with respect to $r$. Thus we can write a Taylor's expansion around $r=r_{0}$ for $f(r)$, using (11) and (12), as follows:

$$
\begin{aligned}
& f(r)=\ln \left[\frac{1}{N} \sum_{i=1}^{N} x_{i}^{r o}\right]+\frac{1}{N} \sum_{i=1}^{N} x_{i}^{r 0}\left(\ln x_{i}\right)\left[\frac{1}{N} \sum_{i=1}^{N} x_{i}^{0}\right]^{-1}\left(r-r_{0}\right) \\
& +\frac{1}{2}\left\{\frac{1}{N} \sum_{i=1}^{N} x_{i}^{r 0}\left(\ln x_{i}\right)^{2}\left[\frac{1}{N} \sum_{i=1}^{N} x_{i}^{r 0}\right]^{-1}\right. \\
& \left.-\left[\frac{1}{N} \sum_{i=1}^{N} x_{i}^{0}\left(\ln x_{i}\right)\right]^{2}\left[\frac{1}{N} \sum_{i=1}^{N} x_{i}^{0}\right]^{-2}\right\}\left(r-r_{0}\right)^{2} \\
& +\frac{1}{6}\left\{\frac{1}{N} \sum_{i=1}^{N} x_{i}^{50}\left(\ln x_{i}\right)^{3}\left[\frac{1}{N} \sum_{i=1}^{N} x_{i}^{50}\right]^{-1}\right. \\
& -\left[3 \frac{1}{N} \sum_{i=1}^{N} x_{i}^{50}\left(\ln x_{i}\right)^{2} \cdot \frac{1}{N} \sum_{i=1}^{N} x_{i}^{50}\left(\ln x_{i}\right)\right]\left[\frac{1}{N} \sum_{i=1}^{N} x_{i}^{50}\right]^{-2} \\
& \left.+2\left[\frac{1}{N} \sum_{i=1}^{N} x_{i}^{r 0}\left(\ln x_{i}\right)\right]^{3}\left[\frac{1}{N} \sum_{i=1}^{N} x_{i}^{r 0}\right]^{-3}\right\}\left(r-r_{0}\right)^{3} \\
& +\ldots \text {. }
\end{aligned}
$$

Putting $r_{0}=0$ and using $(10)$ and $(8),(13)$ becumes

$$
\begin{aligned}
\ln M= & \ln G+\frac{r}{2} \frac{1}{N} \sum_{i=1}^{N}\left(\ln x_{i}-\ln G\right)^{2} \\
& +\frac{r^{2}}{6} \frac{1}{N} \sum_{i=1}^{N}\left(\ln x_{i}-\ln G\right)^{3}+\ldots
\end{aligned}
$$


Setting $r$ equal to unity, (14) yields*

$$
\begin{aligned}
\ln A= & \ln G+\frac{1}{2} \frac{1}{N} \sum_{i=1}^{N}\left(\ln x_{i}-\ln G\right)^{2} \\
& +\frac{1}{6} \frac{1}{N} \sum_{i=1}^{N}\left(\ln x_{i}-\ln G\right)^{3}+\ldots
\end{aligned}
$$

On substituting (15) into (14) and deleting the third and higter order sample moments of $\ln x_{i}(i=1, \ldots, N)$, we obtain the following approximate relationship between the arithmetic and generalized averages

$$
\ln M \approx \ln A+\left(\frac{r-1}{2}\right) \frac{1}{N} \sum_{i=1}^{n}\left(\ln x_{i}-\ln G\right)^{2}
$$

Of course, this approximation is valid only when the sum of the terms containing the third and higher order moments of $\ln x$ converges to a value near zero. Though making a general proposition about this point seems to be impossible, it can at least be shown that (16) holds exactly in a case that frequently turns up in economic discussions, i.e., the case where the variables $x_{1}(i=! \ldots, N)$ are lognormally distributed.

Let us therefore assume that the variables $x_{i}$ are lognormally distributed, i.e.,

$$
f\left(\ln x_{1}\right)=(\sigma \cdot(2 \pi))^{-1} \exp \left\{-\frac{1}{2} \sigma^{-2}\left(\ln x_{l}-\mu\right)^{2}\right\}
$$

We consider

$$
A=E[x]=E[\exp \{\ln x\}]=\exp \left\{\mu+\frac{1}{2} \sigma^{2}\right\}
$$

and we consider the generalized mean $M$,

$$
\begin{aligned}
M & =\left(E\left[x^{\prime}\right]\right)^{1 / r}=\left(E[\exp \{r \ln x\})^{i / r}\right. \\
& =\left(\exp \left\{\mu r+\frac{1}{2} \sigma^{2} r^{2}\right\}\right)^{1 / r}=\exp \left\{\mu+\frac{1}{2} \sigma^{2} r\right\}
\end{aligned}
$$

We then find the exact result, substituting sample moments for theoretical moments.

$$
M=A \exp \left\{\frac{r-1}{2} \frac{1}{N} \sum_{i=1}^{N}\left(\ln x_{i}-\ln G\right)^{2}\right\} .
$$

Going back to the CES production function, we can conclude that we overestimat: our aggregates in using arithmetic averages instead of generalized

'Note that this formula is ver', similar to the one given in Cramer (1971, p. 180). 
averages of the form (5). In case our assumption of lognormality holds true, the relative error is between one and one half times the second-order moment, for values of the elasticity of substitution between 0.5 and 1.0. The error is smaller for the aggregates of value added, if $v>1$, as is commonly found in time series analysis.

Finally, it should be noted that when the second-order moments of the distributions of $\ln K_{t t}$ and $\ln L_{t}$ are equal, the aggregation errors only affect the efficiency parameter $\gamma$; when these second-order moments are different, the aggregation eriors affect the efficiency parameter $\gamma$ as well as the distribution parameter $\delta$, but leave the substitution paranieter $\rho$ and the scale parameter $v$ unaffected.

\section{References}

Bridge, J.L., 1971, Applied econometrics (North-Hollend, Amsterdam).

Cramer, J.S., 1971, Empirical econometrics (No. th-Holland, Amsterdam).

Hardy, G.H., J.E. Littlewoo: and G. Polya, 1967, Inequalities (Cambridge University Press, Cambridge). 